\title{
EGb 761 (Ginkgo biloba) protects cochlear hair cells against ototoxicity induced by gentamicin via reducing reactive oxygen species and nitric oxide-related apoptosis is
}

\author{
Ting Hua Yang ${ }^{\mathrm{a}, \mathrm{b}}$, Yi Ho Young ${ }^{\mathrm{b}}$, Shing Hwa Liü,* \\ a Institute of Toxicology, College of Medicine, National Taiwan University, Taipei 10051, Taiwan \\ ${ }^{\mathrm{b}}$ Department of Otolaryngology, National Taiwan University Hospital, Taipei 10051, Taiwan
}

Received 31 March 2010; received in revised form 29 July 2010; accepted 5 August 2010

\begin{abstract}
Gentamicin is an effective and powerful antibiotic. Extended use or excessive dosages of which can result in irreversible damage to the inner ear. The development of otoprotective strategies is a primary and urgent goal in research of gentamicin ototoxicity. Ginkgo biloba leaves and their extracts are among the most widely used herbal products and/or dietary supplements in the world. We investigated the protection of EGb 761 (a standardized preparation of EGb) on gentamicin ototoxicity and the involvement of reactive oxygen species (ROS) and nitric oxide (NO)-related mechanisms using in vitro organ cultures and an in vivo animal model. Gentamicin induced hair cell damage in cochlear cultures that could be prevented by EGb 761 . EGb 761 also significantly reduced gentamicin-induced ROS and NO production. Furthermore, EGb 761 inhibited cellular apoptosis in cultured cochleae treated with gentamicin. In guinea pigs with gentamicin application onto the round window membrane, the mean auditory brain stem response threshold, ratio of cochlear hair cell damage and apoptosis were significantly elevated compared with those in the control group, and this could be prevented by oral administration of EGb 761 . Individual EGb 761 components quercetin, bilobalide, ginkgolide A and ginkgolide B, but not kaempferol, significantly prevented gentamicin-induced hair cell damage. These results indicate that EGb 761 has a protective effect against gentamicin ototoxicity through a reduction in the formation of ROS and NO and subsequent inhibition of hair cell apoptosis in the cochlea.
\end{abstract}

(c) 2010 Elsevier Inc. All rights reserved.

Keywords: Cell death; Cochlear culture; EGb 761; Hearing; Ototoxicity

\section{Introduction}

Gentamicin, an aminoglycoside antibiotic, is widely used to treat infection caused by Gram-negative bacteria. However, the negative aspect of gentamicin therapy after long-term use refers to its adverse effects, which are mostly nephrotoxicity and ototoxicity. The manifestations of gentamicin-induced ototoxicity consist of hearing loss, tinnitus and vertigo. Since gentamicin is effective, of low cost and broadly used worldwide, gentamicin-induced ototoxicity is widespread. Therefore, the development of otoprotective strategies is a primary and urgent goal in research on gentamicin-induced ototoxicity. Many research studies have suggested that gentamicin-induced ototoxicity is commonly regarded to be mediated by reactive oxygen species (ROS) and reactive nitrogen species [1-6]. Overproduction of ROS triggers signaling pathways of cellular apoptosis, resulting in inner ear damage. Additionally, gentamicin also enhances the formation of nitric oxide (NO) in the inner ear through regulating the inducible NO synthase (iNOS) expression and later forms the

This study was supported by grants from the National Taiwan University Hospital (96-M044 and 97-M1009) and the National Science Council of Taiwan (NSC 96-2628-B-002-058-MY3).

* Corresponding author. Fax: +886 223410217.

E-mail address: shinghwaliu@ntu.edu.tw (S.H. Liu). destructive peroxynitrite with ROS [7]. Several agents that scavenge ROS or block their formation [8-12] or reduce apoptosis via inhibiting various stages of the cell death pathway have been proposed to protect the inner ear [13-17]. Nevertheless, most agents have not yet been used in clinical practice, and the effectiveness of these agents should be weighed against potential hazards before clinical usage.

Ginkgo biloba leaves and their extracts are among the most widely used herbal products and/or dietary supplements in the world. The extract of G. biloba (EGb) has long and safely been used to treat patients with neurodegenerative, vascular and audiovestibular disorders [18-21]. Pharmacologically, EGb is able to modulate the hemodynamics in the cerebrum under normal and ischemic conditions $[22,23]$. In aging rats, EGb treatment has been found to lower circulating free cholesterol and inhibit the production of brain $\beta$ amyloid precursor protein and amyloid $\beta$-peptide [24]. Moreover, EGb has been shown to possess NO-scavenging ability [25,26], which inhibits the expression of iNOS in several tissues [27-29]. A recent study has also shown that EGb prevents the formation of experimental myringosclerosis by scavenging free oxygen radicals [30]. EGb 761 is a standardized formula of EGb that contains terpenoids (6\%, including ginkgolides and bilobalides) and flavonoid glycosides ( $24 \%$, such as kaempferol and quercetin) [31]. These active ingredients in EGb 761 help prevent platelet aggregation and act as potent antioxidants [32-34]. They reacted with several free radicals, 
including superoxide, hydroxyl and peroxyl radicals, to protect tissues from oxidative stress $[35,36]$. Hence, EGb 761 shows promise in ameliorating gentamicin-induced ototoxicity, although its preventive effect and possible mechanism remain to be clarified. Therefore, the aims of this study were to assess the protective effects of EGb 761 on hair cell injury, cell apoptosis and ROS and NO production in cultured cochleae treated with gentamicin and to evaluate the ameliorative effect of EGb 761 on the auditory function in gentamicin-treated animals.

\section{Materials and methods}

\subsection{In vitro organotypic cultures of cochleae}

The cochlea was dissected from Wistar rats on Postnatal Day 3 and cultured based on the methods of Van de Water and Ruben [37] and Sobkowicz et al. [38]. Wistar rats were decapitated on Postnatal Day 3, with their temporal bones removed and tympanic bulla opened. The whole cochlea was carefully dissected out and divided into apical, middle and basal turns in a collagen-coated 35-mm culture dish. The cochlear explants were maintained in Dulbecco's modified Eagle's medium F12 (Gibco, Grand Island, NY, USA) plus $5 \%$ fetal bovine serum, $25 \mathrm{mM}$ Hepes buffer and $30 \mathrm{U} / \mathrm{ml}$ of penicillin. They were incubated at $37^{\circ} \mathrm{C}$ in a humidified atmosphere of $95 \%$ air and $5 \% \mathrm{CO}_{2}$, with the culture medium renewed daily. Initially, EGb 761 was added into the medium at a final concentration of $0,10,20,50,100$ or $200 \mu \mathrm{g} / \mathrm{ml}$ for pretreatment. After $12 \mathrm{~h}$, gentamicin was added into the medium at a final concentration of $0,5,10,20$ or $50 \mu \mathrm{M}$ to induce toxicity. In some experiments, five constituents of EGb 761 (kaempferol, quercetin, bilobalide, ginkgolide A and ginkgolide B) were used to compare their effects with EGb 761 against gentamicin-induced cytotoxicity. These compounds were purchased from Sigma Chemical (St. Louis, MO, USA).

\subsection{In vivo study}

Randomly bred male Hartley strain guinea pigs weighing 200-250 g from an inhouse breeding company were used for in vivo study, housed at $23^{\circ} \mathrm{C} \pm 2{ }^{\circ} \mathrm{C}$ and $55 \%$ $\pm 5 \%$ humidity and given a solid diet of GB- 1 and water ad libitum. For in vivo study, general anesthesia was achieved in guinea pigs by intraperitoneal injection of pentobarbital sodium (35 mg/kg). A small postauricular incision was done, and the tympanic bulla was identified. The bulla was then opened to expose the round window area. Gentamicin $(40 \mathrm{mg} / \mathrm{ml})$ was injected directly overlaying but not through the round window membrane on the left ear. The right ear was injected with $0.05 \mathrm{ml}$ of saline in the same fashion and served as the control. The rationale to select this dosage is based on the amount of $0.3-1.0 \mathrm{ml}(10-80 \mathrm{mg} / \mathrm{ml})$ gentamicin used for humans in clinical practice [39]; thus, one-tenth of the dose was chosen for the guinea pigs [40]. On the other hand, the EGb 761 employed herein was purchased from Dr. Willmar Schwabe Pharmaceuticals (Karlsruhe, Germany); $120 \mathrm{mg}$ of the proprietary concentrated extract of $G$. biloba EGb 761 equivalent to $6 \mathrm{~g}$ of dry leaves standardized to $28.8 \mathrm{mg}$ of ginkgo flavonoid glycosides and $7.2 \mathrm{mg}$ of terpene lactones (3.7 $\mathrm{mg}$ of ginkgolides A, B and C and $3.5 \mathrm{mg}$ of bilobalide) and contained less than $0.5 \mathrm{ppm}$ of ginkgolic acids. Gentamicin was obtained from Sigma Pharmaceuticals (St. Louis, MO, USA). Guinea pigs were orally administered EGb 761 with a dosage of $100 \mathrm{mg} / \mathrm{kg}$ in liquid form once daily for 2 days of pretreatment. On the third day, in addition to EGb 761 feeding, $50 \mu \mathrm{l}$ of gentamicin $(40 \mathrm{mg} / \mathrm{ml})$ was injected intratympanically overlaying the round window membrane. These animals were subjected to auditory brain stem response (ABR) tests and subsequent evaluation of apoptosis in the cochlea. All animal procedures were approved by the institutional review board of the university and were conducted in accordance with the guidelines for the care and use of laboratory animals by the Animal Research Committee of the National Taiwan University College of Medicine.

\subsection{Hair cell staining and counting}

The cochlear organotypic cultures from postnatal rats were prepared for hair cell staining and counting. The cochleae were also divided into three turns and were fixed with $4 \%$ paraformaldehyde and $1 \%$ glutaraldehyde in phosphate-buffered saline (PBS) for $20 \mathrm{~min}$ at $20^{\circ} \mathrm{C}$ and then permeabilized with $5 \%$ Triton X-100 in PBS with $10 \%$ fetal bovine serum for $30 \mathrm{~min}$. These cochlear explants were stained with a conjugated phalloidin-rhodamine probe (1:100, Texas Red-X phalloidin, Molecular Probes, Invitrogen, Carlsbad, CA, USA) in PBS for $1 \mathrm{~h}$. Phalloidin is a specific marker for cellular F-actin and labels stereociliary arrays and cellular borders in the cuticular plate. The tissues were washed three times with PBS and mounted on glass slides in glycerin containing Fluoromount (Molecular Probes, Invitrogen). Finally, the slides were examined via confocal microscopy (Zeiss LSM 510 Meta, Hamburg, Germany). The excitation wavelength was $561 \mathrm{~nm}$, and the emission wavelength was long-passfiltered over $575 \mathrm{~nm}$. Hair cells were counted separately along the apical, middle and basal turns in a cochlea. Depending on the whole length of each turn, five to seven areas (each with a length of $100 \mu \mathrm{m}$ ) were randomly selected for hair cell counting by two independent observers. Counting was repeated in at least three cochleae in each experiment group.

\subsection{Terminal deoxynucleotidyl transferase-mediated dUTP nick end labeling}

Cochleae isolated from guinea pigs and those from postnatal rats were subjected to apoptosis assay. DNA fragmentation of the apoptotic cells was detected by the terminal deoxynucleotidyl transferase-mediated dUTP nick end labeling (TUNEL) technique (DeadEnd Fluorometric TUNEL System, Promega, Madison, WI, USA). One week after gentamicin application onto the round window membrane, the cochleae of the guinea pigs were dissected from the temporal bone after fixation by intracardiac perfusion of $4 \%$ paraformaldehyde and $2.5 \%$ glutaraldehyde in PBS. Cochlear cultures isolated from postnatal rats were also fixed with the same fixatives after treatment. These cochlear explants were incubated in $0.2 \%$ Triton X-100 in PBS for 5 min and then washed twice with PBS. Subsequently, the samples were covered with $100 \mu \mathrm{l}$ of equilibration buffer for 5-10 min at room temperature and then $50 \mu \mathrm{l}$ of terminal deoxynucleotidyl transferase reaction mix containing fluorescein-12-dUTP and recombinant terminal deoxynucleotidyl transferase enzyme was added for 60 -min incubation at $37^{\circ} \mathrm{C}$ in the dark. Rhodamine-phalloidin probe was used to label hair cells and DAPI to counterstain nuclei of normal or apoptotic cells. Finally, the cochlear explants were mounted and photographed with a confocal microscope. The excitation wavelengths to observe TUNEL stain and DAPI were 488 and $405 \mathrm{~nm}$, respectively, and the emission wavelengths were 505-550 and $420-480 \mathrm{~nm}$, respectively. Deoxyribonuclease I (DNase I) was used as a positive control, which generates strand breaks in the DNA to provide a positive TUNEL reaction.

\subsection{Measurement of ROS in the cochlea}

The cell-permeant dye $\mathrm{H}_{2}$ DCF-DA is the reduced and acetylated form of $2^{\prime}, 7^{\prime}$ dichlorofluorescein (DCF). Esterase cleavage of the lipophilic blocking groups yields a charged form of the dye that is much better retained by cells than is the parent compound. ROS oxidizes $\mathrm{H}_{2} \mathrm{DCF}$ to the fluorescent compound DCF, which produces bright green fluorescence. We used carboxy- $\mathrm{H}_{2} \mathrm{DCF}-\mathrm{DA}$ (Invitrogen), the carboxy derivative of $\mathrm{H}_{2}$ DCF-DA, to evaluate the production of ROS in the cochlea. Carboxy$\mathrm{H}_{2}$ DCF-DA carries additional negative charges that improve its retention compared with noncarboxylated forms. The experiment followed the method of Trayner et al. [41] with some modifications. Briefly, cochlear organotypic culture receiving a different treatment was planted in a 96-well microplate (Corning Incorporated, Corning, NY, USA). Each treatment group was composed of eight cochlear explants pooled together from four postnatal rats. After specific treatment in each group, these cochlear explants were washed with PBS and then incubated with $10 \mathrm{mM}$ carboxy- $\mathrm{H}_{2}$ DCF-DA for $30 \mathrm{~min}$ at $37^{\circ} \mathrm{C}$ in a humidified atmosphere of $95 \%$ air and $5 \% \mathrm{CO}_{2}$. After $30 \mathrm{~min}$, the extracellular ROS dye was washed away with Dulbecco's modified Eagle's medium F12 and the microplate was then put in a microplate reader (DTX 800 Multimode Detector, Beckman Coulter, Fullerton, CA, USA) to have the fluorescence read. The excitation and emission wavelengths used were 485 and $538 \mathrm{~nm}$, respectively.

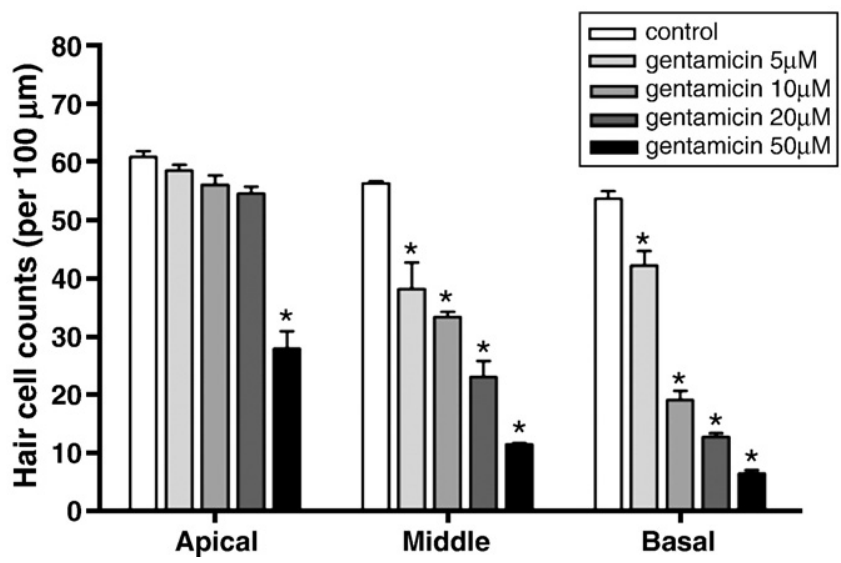

Fig. 1. Dose-dependent cytotoxicity of gentamicin on the hair cells of organotypic cochlear cultures of postnatal rats. The organ of Corti from postnatal 3-day-old rats was incubated in medium containing 0-50 $\mu \mathrm{M}$ gentamicin for $48 \mathrm{~h}$. Labeled with rhodamine-phalloidin, hair cells were counted under a confocal microscope, and their number $/ 100 \mu \mathrm{m}$ was averaged separately along the apical, middle and basal turns in cochleae. Gentamicin $(50 \mu \mathrm{M})$ effectively produced global cytotoxicity in the apical, middle and basal turns of the cochleae. Data are presented as the mean \pm S.D. ${ }^{*} P<.05$, when compared with the respective control group. 


\subsection{Measurement of NO in the cochlea}

Much of the NO in tissue is oxidized to nitrite and nitrate, and the concentrations of these anions have been used as a quantitative measure of NO production. A Total Nitric Oxide Assay Kit (Assay Designs, Ann Arbor, MI, USA) was used to determine total nitrite/nitrate in the cochlea. This involves the conversion of nitrate to nitrite by nitrate reductase, followed by the colorimetric detection of nitrite as a colored azo dye product of the Griess reaction that absorbs visible light at $540 \mathrm{~nm}$. The organotypic cochlear culture of postnatal rats was processed to determine the level of NO. Cochleae from four rats in each group were pooled together to increase detectability of the assay. The cochlear explants were frozen with liquid nitrogen and then pulverized into a powder using a pellet pestle rod with microtube (Kimble/Kontes, Vineland, NJ, USA) and homogenized in ice-cold 50 $\mathrm{mM}$ Tris-EDTA buffer ( $\mathrm{pH}$ 7.0). The homogenate was centrifuged at 10,000g for 45 min at $4^{\circ} \mathrm{C}$. The supernatant was used to determine the levels of NO metabolites. Nitrate reductase was added to each sample to convert nitrate to nitrite. These samples were incubated with enzyme solution for $30 \mathrm{~min}$ at $37^{\circ} \mathrm{C}$ before Griess reagent was added. Their absorbance at $540 \mathrm{~nm}$ was read on a microplate reader.

\subsection{ABRs}

Two weeks after gentamicin application in the ear, each guinea pig underwent an $\mathrm{ABR}$ test to evaluate hearing status. After anesthesia with intraperitoneal pentobarbital sodium, a pair of needle electrodes was placed onto the vertex and the ipsilateral retroauricular region, while a ground electrode was placed on the neck. Click stimuli were delivered through a plastic tube inserted into the ear canal (Smart EP2, Intelligent Hearing Systems, Miami, FL, USA). The repetition rate was 57.7/s, and 1000 sweeps were averaged. The stimulus intensity was from $100 \mathrm{~dB}$ SPL initially, followed by 10 - to 5-dB step decrements until the absence of waveforms, and the threshold of ABR was thus determined. The mean threshold of $A B R$ was calculated from 10 guinea pigs in each group.
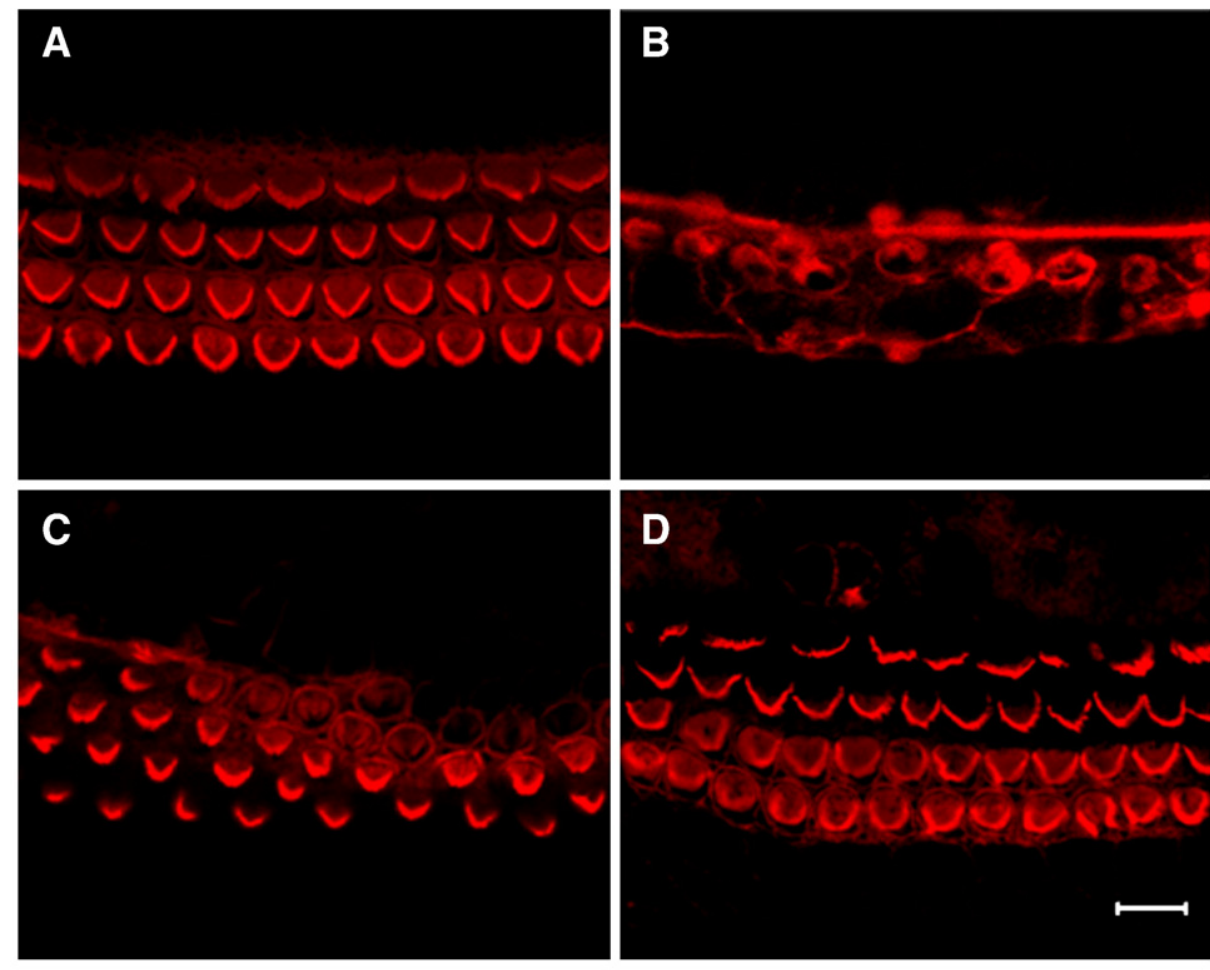

\section{E}

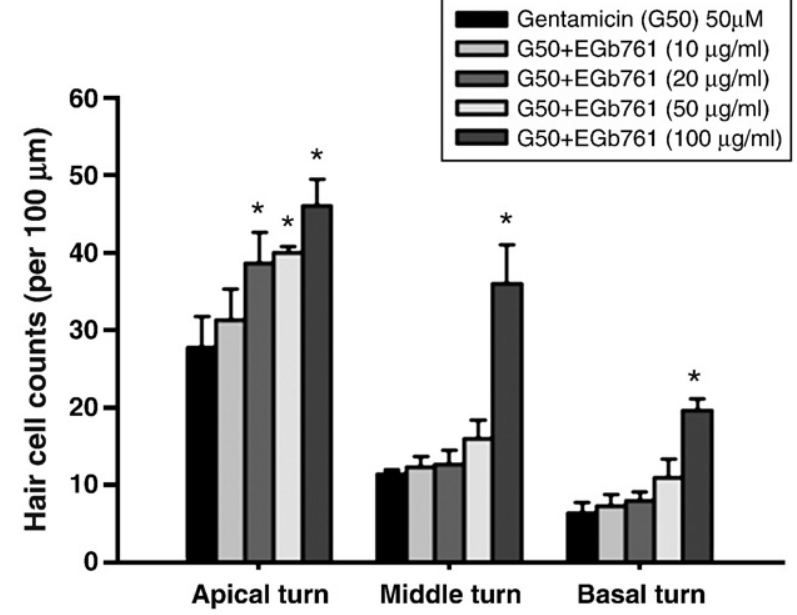

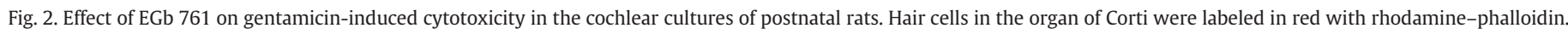

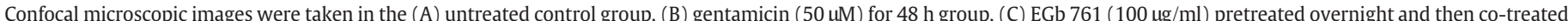

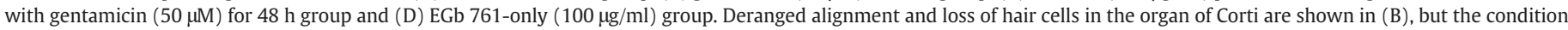

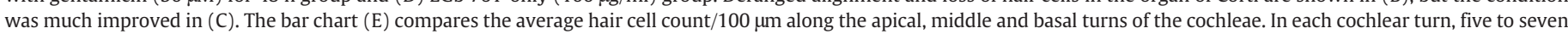

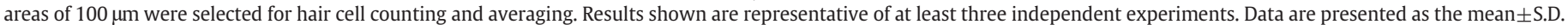
${ }^{*} P<.05$, as compared with the gentamicin group. The scale bar represents $10 \mu \mathrm{m}$. 


\subsection{Statistical analysis}

The average hair cell number is represented as the mean \pm S.D. One-way ANOVA or post hoc test for different interactions within one test was used to analyze statistical significance of the results. The mean ABR thresholds were compared by unpaired twotailed Student's $t$ test. A value of $P<.05$ indicated statistical significance.

\section{Results}

3.1. In vitro study in rat cochlear cultures: gentamicin-induced hair cell damage

Organotypic cultures of the cochlea isolated from postnatal rats were incubated in the medium with $5-50 \mu \mathrm{M}$ gentamicin added for $48 \mathrm{~h}$. In the control group, three rows of outer hair cells and one row of inner hair cells kept their shape, with the stereocilia well preserved. In the gentamicin group, viable hair cells were decreased in number (Fig. 1), and they also lost normal contour and alignment (Fig. 2B). The average hair cell count declined significantly in the middle and basal turns despite the gentamicin concentration being varied from 5 to $50 \mu \mathrm{M}$. However, for the apical hair cells, a significant difference existed only between the control and $50 \mu \mathrm{M}$ gentamicin groups (Fig. 1). Because global hair cell loss of the rat cochlea occurred in the $50 \mu \mathrm{M}$ gentamicin group, the latter was used to evaluate the effect of EGb 761 on gentamicin cytotoxicity.

\subsection{In vitro study in rat cochlear cultures: effect of EGb 761 on} gentamicin cytotoxicity and apoptosis

Twelve hours before gentamicin treatment, cochlear cultures of postnatal rats were incubated in the medium containing EGb 761 at
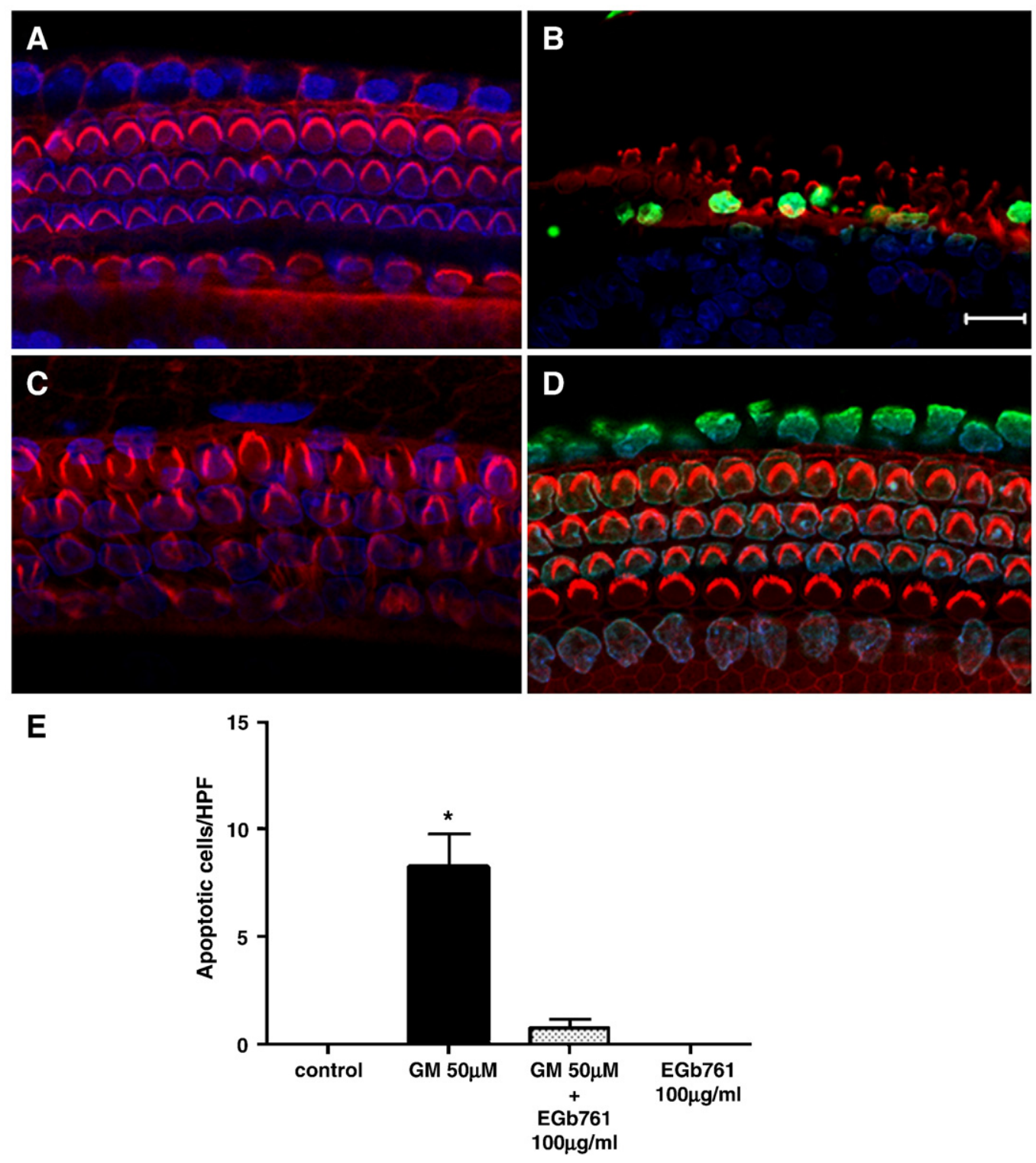

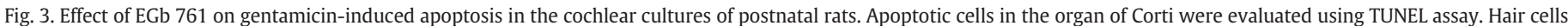

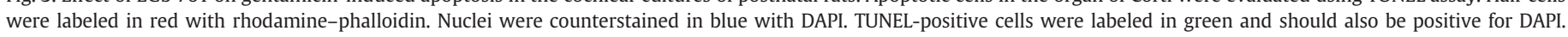

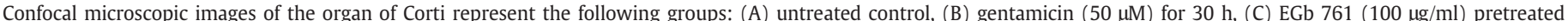

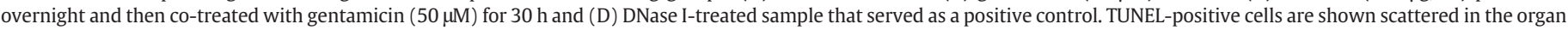

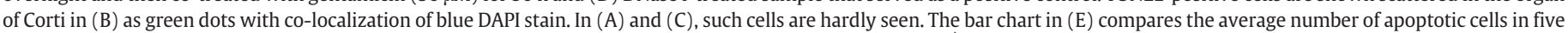

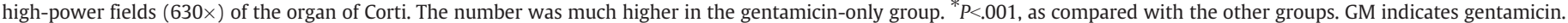
Results shown are representative of three independent experiments. The scale bar represents $10 \mu \mathrm{m}$. 
concentrations of $10-100 \mu \mathrm{g} / \mathrm{ml}$. Viable phalloidin-labeled hair cells were counted after $48 \mathrm{~h}$ of gentamicin $(50 \mu \mathrm{M})$ treatment. Compared with untreated controls (Fig. 2A), gentamicin severely distorted the anatomy of the organ of Corti in the $50 \mu \mathrm{M}$ gentamicin group. Nonviable hair cells were deformed, deranged and not even recognizable (Fig. 2B). EGb 761 was not toxic to the cochlea (Fig. 2D) and reduced gentamicininduced cytotoxicity (Fig. 2C); the alignment of cells in the organ of Corti was somewhat irregular, but hair cells retained their shape and stereocilia (Fig. 2C). The preventive effect was more prominent in the apical turn in a dose-dependent manner (20-100 $\mu \mathrm{g} / \mathrm{ml}$ ) (Fig. 2E). In the middle and basal turns, $100 \mu \mathrm{g} / \mathrm{ml}$ of EGb 761 effectively provided protection against gentamicin (Fig. 2E). Apoptosis was evaluated by TUNEL assay, which stained DNA fragments in the apoptotic cells. TUNEL-positive (apoptotic) cells were found in the cochlear culture after $30 \mathrm{~h}$ of gentamicin $(50 \mu \mathrm{M})$ incubation (Fig. 3B). Pretreatment with EGb $761(100 \mu \mathrm{g} / \mathrm{ml})$ could significantly reduce the number of TUNEL-positive cells in the gentamicin-treated cochlea (Fig. 3C). In the control (Fig. 3A) and EGb 761-only (data not shown) groups, apoptotic cells were not observed. DNase I-treated cochleae served as positive controls of TUNEL staining (Fig. 3D).

\subsection{In vitro study in rat cochlear cultures: EGb 761 reduced gentamicin-induced ROS and NO production}

ROS production between the different treatment groups was compared by reading the fluorescence of DCF. The fluorescence was assessed every 9 min consecutively for $3 \mathrm{~h}$, and the value is presented as fluorescence intensity. Evaluation of ROS levels started after $1 \mathrm{~h}$ of gentamicin incubation. Gentamicin $(50 \mu \mathrm{M})$ elevated ROS levels significantly (Fig. 4). Administration of $100 \mu \mathrm{g} / \mathrm{ml}$ of EGb 761 effectively reduced ROS production to about the same level as that in the control cochleae. EGb 761 alone decreased ROS levels even further. The ROS level in each group was consistently raised during the whole test period as time passed, except for the EGb 761-only group (Fig. 4). A similar trend was also observed in NO production. Gentamicin $(50 \mu \mathrm{M})$ significantly increased NO levels in the cochleae. Administration of EGb 761 reduced NO level to the same level as that in the untreated cochleae (Fig. 5).

\subsection{In vivo study in guinea pigs: auditory function and hair cell damage in groups with gentamicin and/or EGb 761}

Auditory function of the animals was evaluated by determining the ABR threshold. One week after gentamicin treatment, the averaged $A B R$ threshold of gentamicin-treated ears was significantly higher than that of saline-treated control ears (Fig. 6B and E). With oral administration of EGb $761(100 \mathrm{mg} / \mathrm{kg}$ per day) to the gentamicintreated guinea pigs, the ABR threshold was reduced to a level not different from that in the control and EGb 761-only groups (Fig. 6A, C and $\mathrm{D}$ ). Meanwhile, hair cell damage was evident in the cochleae of gentamicin-treated ears compared with the saline controls (Fig. 7B). However, if the animals were treated with daily EGb 761 at $100 \mathrm{mg} / \mathrm{kg}$, hair cell damage was no longer seen in the cochleae (Fig. 7C), similarly as observed in the control and EGb 761-only groups. Likewise, the TUNEL-positive (apoptotic) cells in the organ of Corti were markedly increased in the gentamicin-treated group (Fig. 7B) but were hardly observed in either the controls or the EGb 761-fed guinea pigs with gentamicin treatment (Fig. 7A and C). DNase I-treated cochleae served as positive controls of TUNEL staining (Fig. 7D).

\subsection{Effects of active constituents of EGb 761 on gentamicin cochleotoxicity in rat cochlear cultures}

The effects of kaempferol, quercetin, bilobalide, ginkgolide A and ginkgolide B on gentamicin cochleotoxicity were evaluated as compared

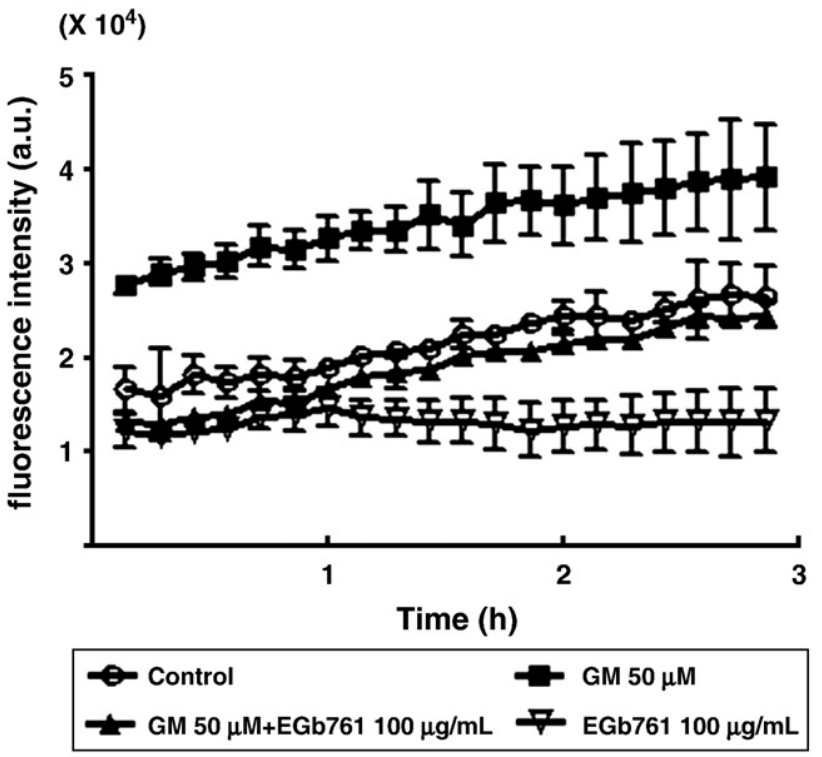

Fig. 4. Effect of EGb 761 on gentamicin-induced ROS levels in the cochlear cultures of postnatal rats. Fluorescence of carboxy- $\mathrm{H}_{2}$ DCF-DA (ROS indicator) from cochlear explants was read every 9 min consecutively for $3 \mathrm{~h}$. Change of fluorescence intensity (a.u.) with time was plotted as a line chart. Four groups were compared: untreated control, gentamicin $(50 \mu \mathrm{M})$ for $1 \mathrm{~h}$, EGb $761(100 \mu \mathrm{g} / \mathrm{ml})$ overnight plus gentamicin $(50 \mu \mathrm{M})$ for $1 \mathrm{~h}$ and EGb 761 only. The ROS level in the gentamicin-only group was significantly elevated compared with the other groups $(P<.001)$. The ROS level in the EGb 761 group with or without gentamicin was not different from that in the control group statistically $(P>.05)$. Data are presented as the mean \pm S.D. Results shown are representative of three independent experiments.

with EGb 761. Twelve hours before gentamicin treatment, cochlear cultures of postnatal rats were incubated in medium containing kaempferol, quercetin, bilobalide, ginkgolide $\mathrm{A}$ and ginkgolide $\mathrm{B}$ at concentrations of $0.3-100 \mu \mathrm{g} / \mathrm{ml}$. Viable phalloidin-labeled hair cells were counted after $48 \mathrm{~h}$ of gentamicin $(50 \mu \mathrm{M})$ treatment. Quercetin, bilobalide, ginkgolide A and ginkgolide B, but not kaempferol, significantly prevented gentamicin-induced hair cell damage (Fig. 8).

\section{Discussion}

Hair cells are the most vulnerable element in the cochlea to gentamicin toxicity and were thus chosen to be an indicator of

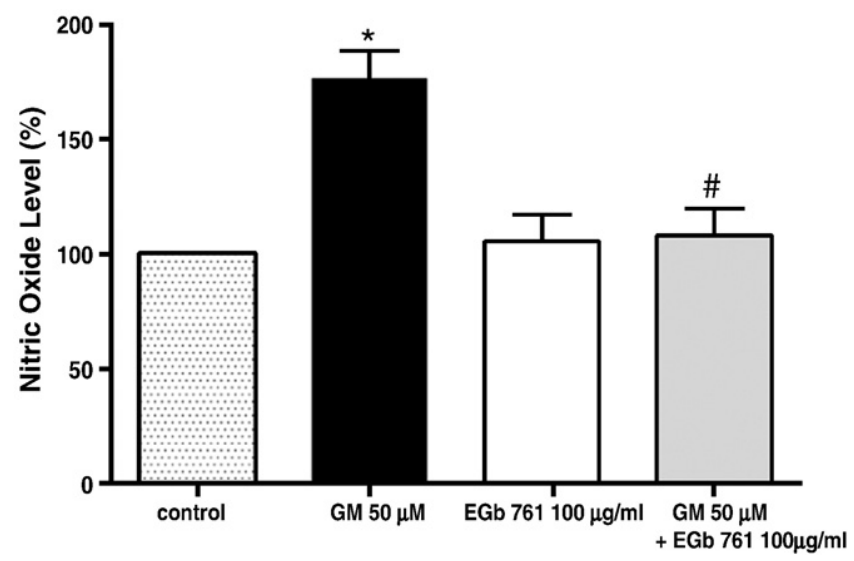

Fig. 5. Effect of EGb 761 on gentamicin-induced NO production in the cochlear cultures of postnatal rats. NO production in cochlear culture incubated with gentamicin $(50 \mu \mathrm{M})$ for $3 \mathrm{~h}$ in the presence or absence of EGb $761(100 \mu \mathrm{g} / \mathrm{ml})$ was quantified and plotted as a bar chart. ${ }^{*} P<.05$, as compared with the control group. ${ }^{\#} P<.05$, as compared with the gentamicin group. Data are presented as the mean \pm S.D. 


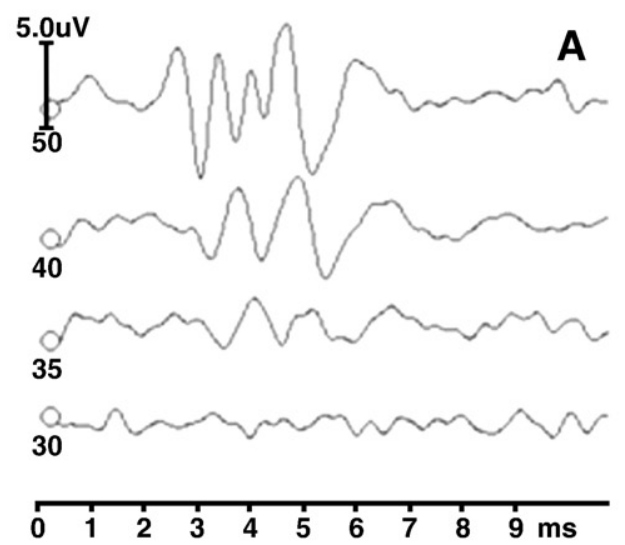

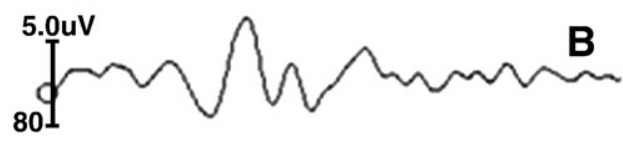

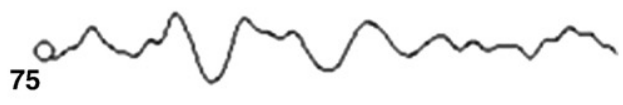

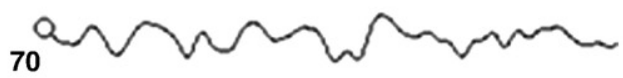

65
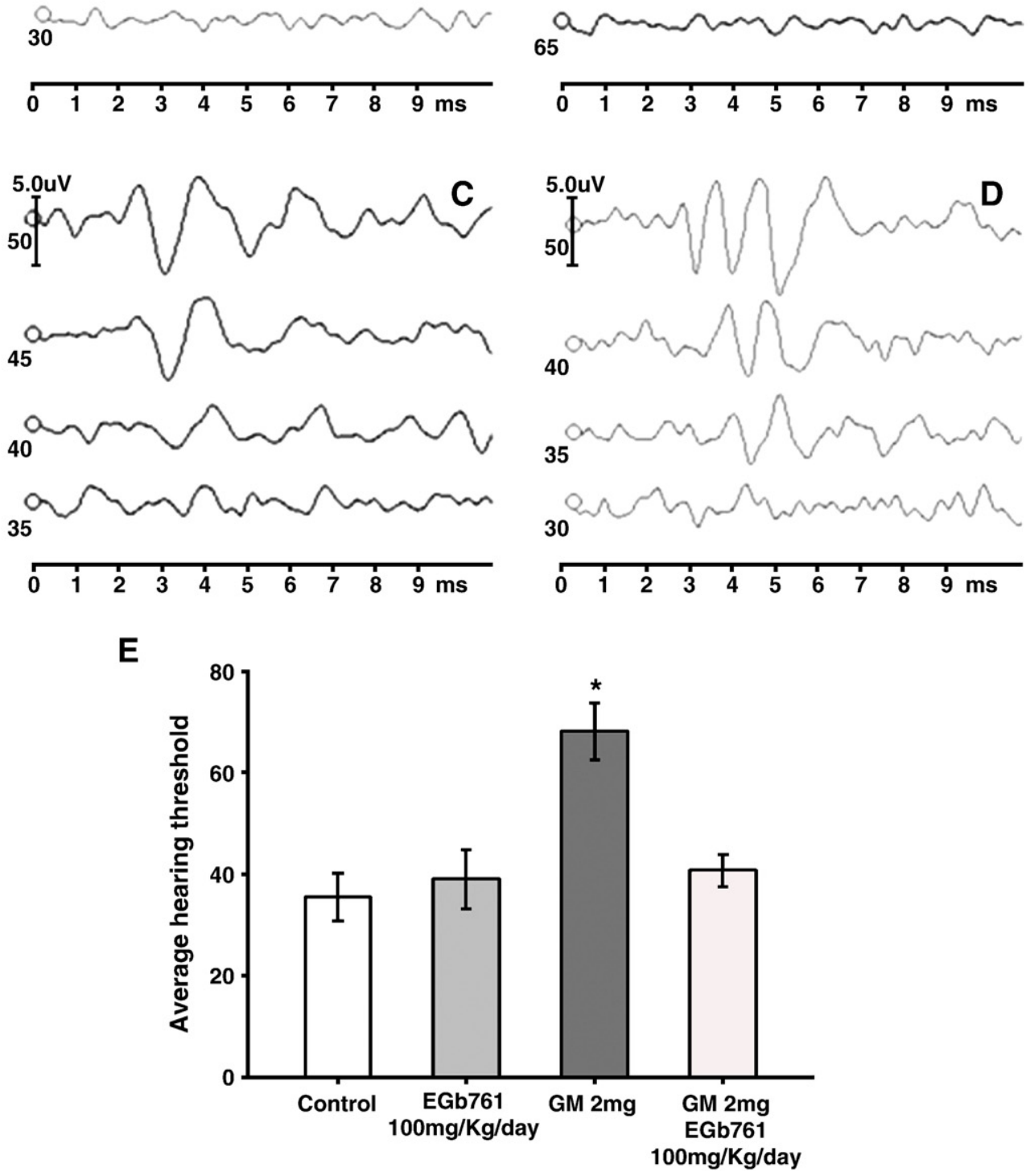

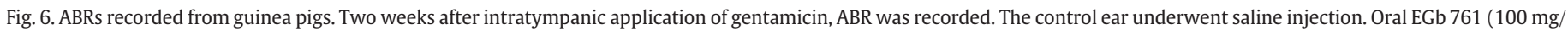

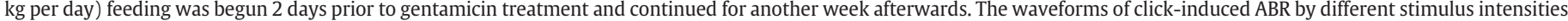

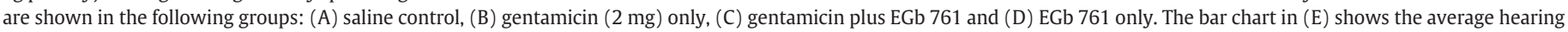

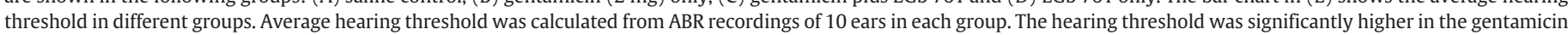

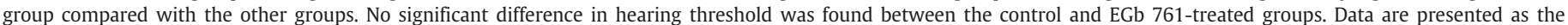
mean \pm S.D. ${ }^{*} P<.005$, as compared with the other groups.

gentamicin cytotoxicity in this study. In this work, at a low concentration of gentamicin $(5 \mu \mathrm{M})$, significant hair cell loss was already observed in the middle and basal turns, while most hair cells of the apical turn remained intact. When the gentamicin concentration was elevated to $50 \mu \mathrm{M}$, all cochlear turns showed a significant decrease in hair cell counts. Thus, in this study, gentamicin at a concentration of $50 \mu \mathrm{M}$, which was adopted to produce global toxicity of the cochleae, was used to evaluate the ameliorated effect of EGb 761 on gentamicin ototoxicity. On the other hand, apical hair cells apparently tolerated a higher concentration of gentamicin, while the hair cells in the basal turn were more susceptible to noxious damage than those in the apical turn. Likewise, clinical patients with drug-induced ototoxicity very often present with early high-frequency sensorineural 

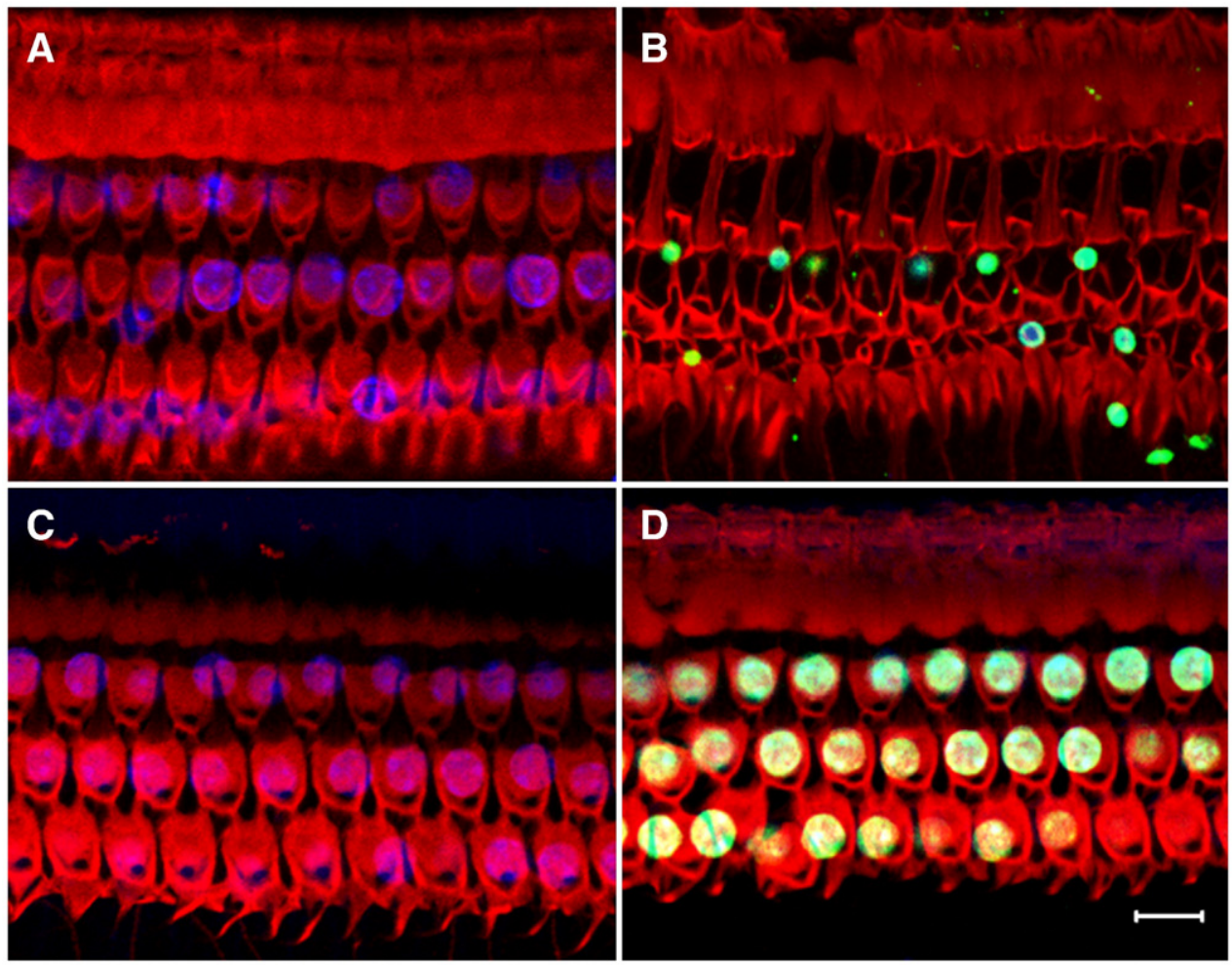

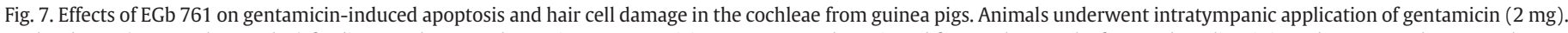

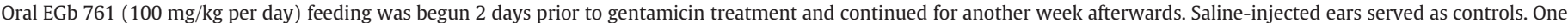

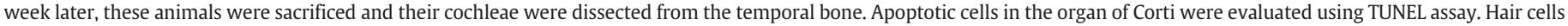

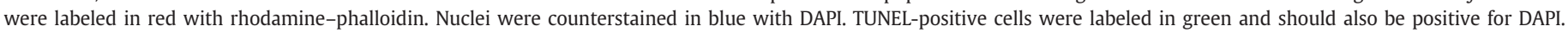

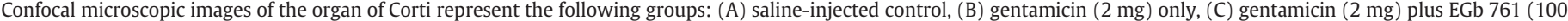

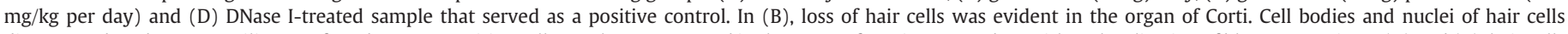

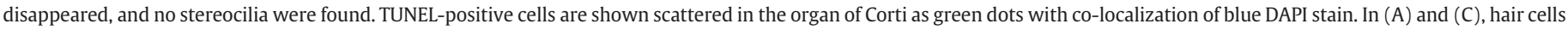
retained a normal shape and no TUNEL-positive cells were seen. Results shown are representative of three independent experiments. The scale bar represents $10 \mu \mathrm{m}$.

hearing loss. Is variation of toxicity in each cochlear turn attributable to the concentration gradient of the drug from base to apex? The present study found otherwise, since each turn was exposed to the same concentration of gentamicin in an organotypic cochlear culture system. Moreover, Sha et al. [42] have reported that basal outer hair cells display a lower level of glutathione and died earlier than apical ones in an untreated cochlear culture of guinea pigs. Thus, the intrinsic difference of the hair cells in various cochlear regions should be responsible for their dissimilar tolerance to gentamicin insult.

Overproduction of ROS was suggested to be an initial step to trigger apoptotic pathways and cause cell death in aminoglycosideinduced ototoxicity. The methods of blocking ROS in the cochlea under in vitro or in vivo aminoglycoside exposure have been shown in many studies $[3,43,44]$. NO is also involved in gentamicin ototoxicity. Peroxynitrite, formed by NO together with superoxide, is more reactive and toxic and plays an important role in gentamicin-induced ototoxicity [5]. Recent in vivo studies have shown that gentamicin is capable of inducing up-regulation of iNOS [45] and endothelial NO synthase [46] expression in the cochlea of guinea pigs. Heinrich et al. [1] discovered the correlation between hearing threshold shift and NO production in the cochlea and concluded that increased NO contributed to gentamicin-induced hearing loss. In this work, we found that increased levels of ROS and NO production were evident after gentamicin treatment in the cochlear cultures and that EGb 761 could effectively reduce ROS and NO production to a level near or even lower than that in the untreated cochleae. Therefore, these findings suggest that EGb 761 possesses the ability to antagonize gentamicin-induced ototoxicity possibly via inhibiting ROS and NO formation.

Our in vivo study on guinea pigs has demonstrated that EGb 761 prevents apoptosis and hair cell loss in the cochlea and consequently protects against gentamicin-induced impaired auditory function. EGb has also been suggested to improve blood flow or microcirculation [47]. Didier et al. [19] have reported that vasodilatation of the cochlear vessels in the spiral lamina is observed in guinea pigs after 6 weeks of treatment with EGb 761. Therefore, it is possible that, in addition to reduction of gentamicin-induced oxidative stress, the hemodynamic modulatory effect of EGb 761 may ameliorate the microenvironment of hair cells and improve hearing status under gentamicin exposure. Moreover, the incomplete recovery of hair cell loss in the in vitro cochlear cultures might be attributed to the prolonged exposure of tissue in a high concentration of gentamicin, unlike the transiently increased concentration in the cochlear perilymph in the gentamicin-treated animals. These findings imply that the strategy on the limitation of ROS and NO production may not be sufficient to prevent gentamicin ototoxicity.

Hyun et al. [48] have demonstrated that 6-hydroxykynurenic acid and other flavonoids obtained from yellow leaves of $\mathrm{G}$. biloba evidenced a marked scavenging activity on authentic ONOO-. It has also been suggested that kaempferol and quercetin, two components of EGb 761, effectively induce caspase-3-dependent apoptosis of oral cavity cancer cells and can be considered as possible antioral cavity cancer agents [49]. In this study, we tested the effects of five constituents of EGb 761 on gentamicin-induced cochleotoxicity in cochlear culture. As suggested in previous reports [50-52] and in 

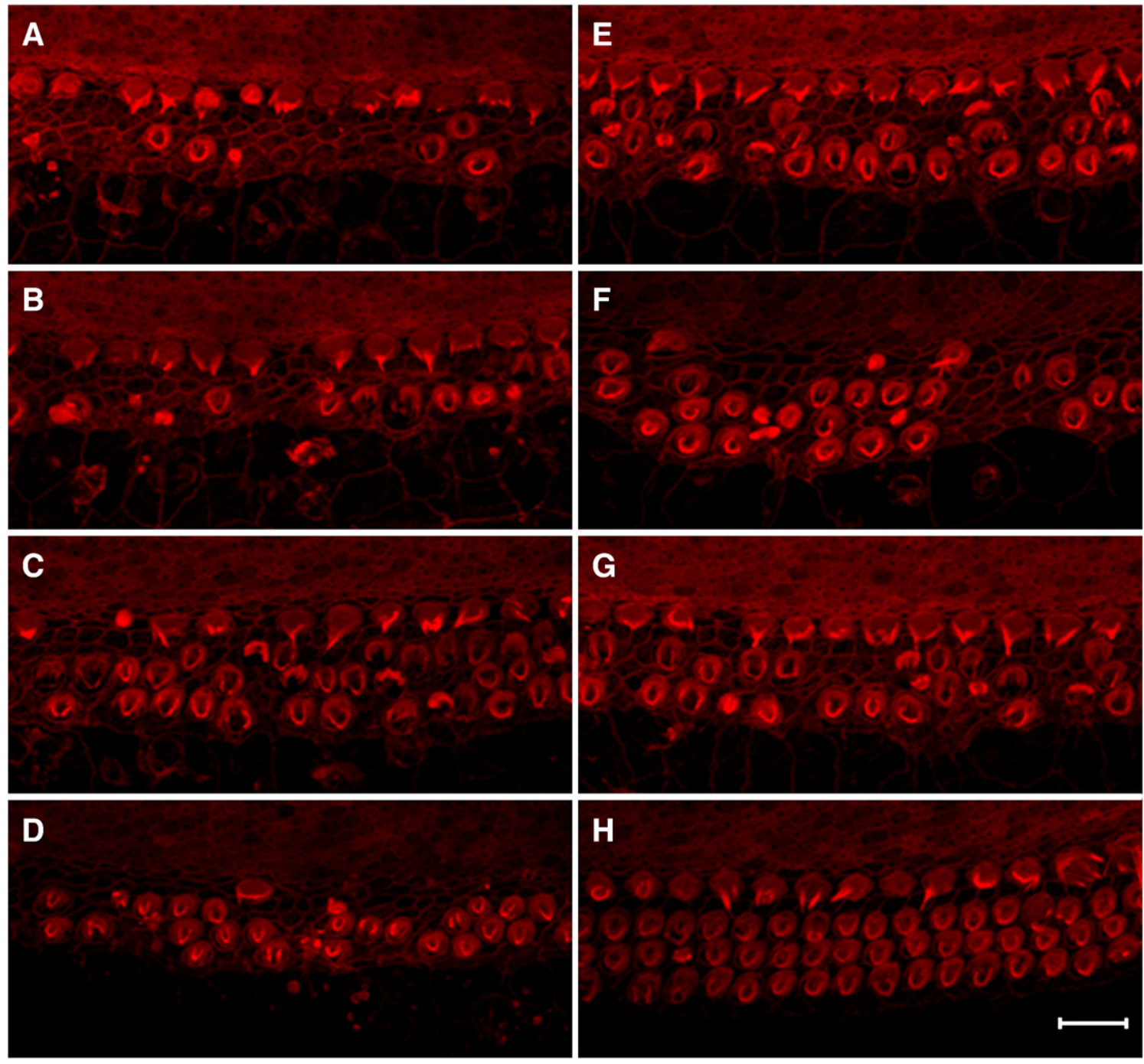

I

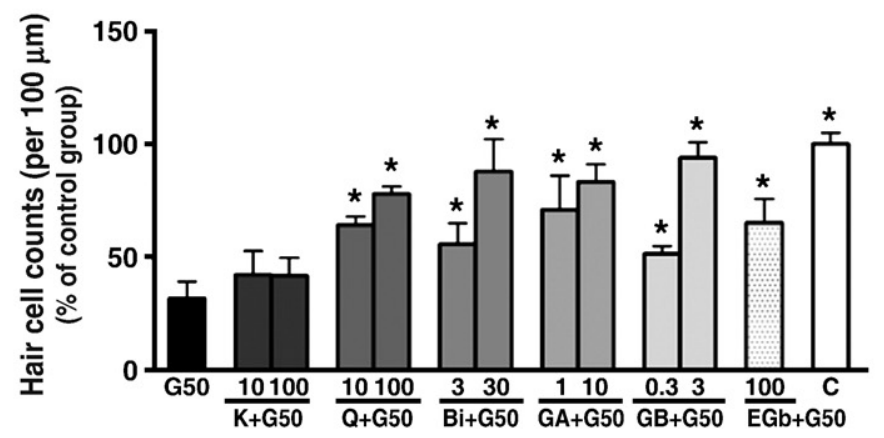

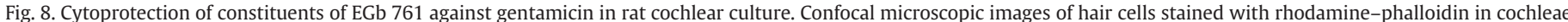

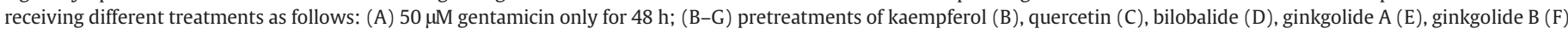

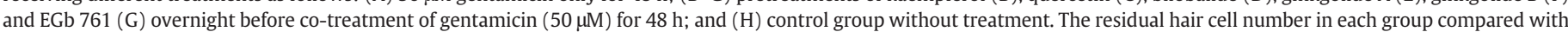

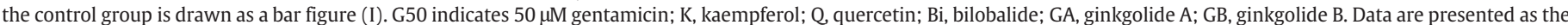
mean \pm S.D. $(n=4) .{ }^{*} P<.05$, as compared with the gentamicin-only group. The scale bar represents $10 \mu \mathrm{m}$.

the EGb 761 datasheet of Dr. Willmar Schwabe Pharmaceuticals, the average levels of kaempferol, quercetin, bilobalide, ginkgolide $\mathrm{A}$ and ginkgolide B in EGb were around 10\%, 10\%, 3\%, 1\% and $0.3 \%$, respectively. Consequently, the concentrations of these constituents in EGb $761(100 \mu \mathrm{g} / \mathrm{ml})$ became 10, 10, 3, 1 and $0.3 \mu \mathrm{g} / \mathrm{ml}$, respectively. Among them, quercetin, bilobalide, ginkgolide $\mathrm{A}$ and ginkgolide B were all able to rescue hair cells against gentamicin. Kaempferol, however, did not show such protection even at high concentration. The results suggest that the two major types of constituents (terpene trilactones and flavonoid glycosides) in EGb 761 are responsible for the protective effect of EGb 716 against gentamicin-induced cochleotoxicity.

In conclusion, EGb 761 possesses a protective effect against gentamicin ototoxicity in the cochlea. EGb 761 attenuates gentamicin cytotoxicity via reducing the formation of ROS and NO, subsequently inhibiting apoptosis of cochlear hair cells. Consequently, EGb 761 can 
preserve more cochlear hair cells and improve hearing status in gentamicin-treated guinea pigs. Our findings suggest that EGb as a dietary supplement/healthy food may possess the potential to prevent gentamicin ototoxicity.

\section{References}

[1] Heinrich UR, Helling K, Sifferath M, Brieger J, Li H, Schmidtmann I, et al. Gentamicin increases nitric oxide production and induces hearing loss in guinea pigs. Laryngoscope 2008;118:1438-42.

[2] López-González MA, Lucas M, Delgado F, Diaz P. The production of free oxygen radicals and nitric oxide in the rat cochlea. Neurochem Int 1998;33:55-9.

[3] Rizzi MD, Hirose K. Aminoglycoside ototoxicity. Curr Opin Otolaryngol Head Neck Surg 2007;15:352-7.

[4] Sha SH. Stimulation of free radical formation by aminoglycoside antibiotics. Hear Res 1999;128:112-8.

[5] Takumida M, Popa R, Anniko M. Free radicals in the guinea pig inner ear following gentamicin exposure. ORL J Otorhinolaryngol Relat Spec 1999;61:63-70.

[6] Wu WJ, Sha SH, Schacht J. Recent advances in understanding aminoglycoside ototoxicity and its prevention. Audiol Neurootol 2002;7:171-4.

[7] Takumida M, Anniko M. Nitric oxide in guinea pig vestibular sensory cells following gentamicin exposure in vitro. Acta Otolaryngol 2001;121:346-50.

[8] Fetoni AR, Sergi B, Ferraresi A, Paludetti G, Troiani D. alpha-Tocopherol protective effects on gentamicin ototoxicity: an experimental study. Int J Audiol 2004;43: 166-71.

[9] Jung HW, Chang SO, Kim CS, Rhee CS, Lim DH. Effects of Ginkgo biloba extract on the cochlear damage induced by local gentamicin installation in guinea pigs. J Korean Med Sci 1998;13:525-8.

[10] McFadden SL, Ding D, Salvemini D, Salvi RJ. M40403, a superoxide dismutase mimetic, protects cochlear hair cells from gentamicin, but not cisplatin toxicity. Toxicol Appl Pharmacol 2003;186:46-54

[11] Sha SH, Schacht J. Antioxidants attenuate gentamicin-induced free radical formation in vitro and ototoxicity in vivo: D-methionine is a potential protectant. Hear Res 2000;142:34-7.

[12] Wang AM, Sha SH, Lesniak W, Schacht J. Tanshinone (Salviae miltiorrhizae extract) preparations attenuate aminoglycoside-induced free radical formation in vitro and ototoxicity in vivo. Antimicrob Agents Chemother 2003;47: $1836-41$.

[13] Bodmer D, Brors D, Pak K, Gloddek B, Ryan A. Rescue of auditory hair cells from aminoglycoside toxicity by Clostridium difficile toxin B, an inhibitor of the small GTPases Rho/Rac/Cdc42. Hear Res 2002;172:81-6.

[14] Corbacella E, Lanzoni I, Ding D, Previati M, Salvi R. Minocycline attenuates gentamicin induced hair cell loss in neonatal cochlear cultures. Hear Res 2004:197:11-8.

[15] Wang J, Van De Water TR, Bonny C, de Ribaupierre F, Puel JL, Zine A. A peptide inhibitor of C-Jun $\mathrm{N}$-terminal kinase protects against both aminoglycoside and acoustic trauma-induced auditory hair cell death and hearing loss. J Neurosci 2003:23:8596-607.

[16] Wei X, Zhao L, Liu J, Dodel RC, Farlow MR, Du Y. Minocycline prevents gentamicininduced ototoxicity by inhibiting p38 MAP kinase phosphorylation and caspase 3 activation. Neuroscience 2005;131:513-21.

[17] Ylikoski J, Xing-Qun L, Virkkala J, Pirvola U. Blockade of c-Jun N-terminal kinase pathway attenuates gentamicin-induced cochlear and vestibular hair cell death. Hear Res 2002;166:33-4.

[18] Burschka MA, Hassan HA, Reineke T, van Bebber L, Caird DM, Mösges R. Effect of treatment with Ginkgo biloba extract EGb 761 (oral) on unilateral idiopathic sudden hearing loss in a prospective randomized double-blind study of 106 outpatients. Eur Arch Otorhinolaryngol 2001;258:213-9.

[19] Didier A, Droy-Lefaix MT, Aurousseau C, Cazals Y. Effects of Ginkgo biloba extract (EGb 761) on cochlear vasculature in the guinea pig: morphometric measurements and laser Doppler flowmetry. Eur Arch Otorhinolaryngol 1996;253: 25-30.

[20] Krieglstein J, Beck T, Seibert A. Influence of an extract of Ginkgo biloba on cerebral blood flow and metabolism. Life Sci 1986;39:2327-34.

[21] Smith PF, Zheng Y, Darlington CL. Ginkgo biloba extracts for tinnitus: more hype than hope? J Ethnopharmacol 2005;100:95-9.

[22] Oberpichler H, Beck T, Abdel-Rahman MM, Bielenberg GW, Krieglstein J. Effects of Ginkgo biloba constituents related to protection against brain damage caused by hypoxia. Pharmacol Res Commun 1988;20:349-68.

[23] Zhang J, Fu S, Liu S, Mao T, Xiu R. The therapeutic effect of Ginkgo biloba extract in SHR rats and its possible mechanisms based on cerebral microvascular flow and vasomotion. Clin Hemorheol Microcirc 2000;23:133-8.

[24] Yao ZX, Han Z, Drieu K, Papadopoulos V. Ginkgo biloba extract (EGb 761) inhibits $\beta$-amyloid production by lowering free cholesterol levels. J Nutr Biochem 2004;15:749-56.
[25] Kobuchi H, Droy-Lefaix MT, Christen Y, Packer L. Ginkgo biloba extract (EGb 761): inhibitory effect on nitric oxide production in the macrophage cell line RAW 264.7. Biochem Pharmacol 1997;53:897-903.

[26] Marcocci L, Maguire JJ, Droy-Lefaix MT, Packer L. The nitric oxide-scavenging properties of Ginkgo biloba extract EGb 761. Biochem Biophys Res Commun 1994;201:748-55.

[27] Cheung F, Siow YL, Chen WZ, O K. Inhibitory effect of Ginkgo biloba extract on the expression of inducible nitric oxide synthase in endothelial cell. Biochem Pharmacol 1999;58:1665-73.

[28] Punkt K, Zaitsev S, Park JK, Wellner M, Buchwalow IB. Nitric oxide synthase isoforms I, III and protein kinase-Ctheta in skeletal muscle fibres of normal and streptozotocin-induced diabetic rats with and without Ginkgo biloba extract treatment. Histochem J 2001;33:213-9.

[29] Shen J, Wang J, Zhao B, Hou J, Gao T, Xin W. Effects of EGb 761 on nitric oxide and oxygen free radicals, myocardial damage and arrhythmia in ischemia-reperfusion injury in vivo. Biochim Biophys Acta 1998;1406:228-36.

[30] Kaptan ZK, Emir H, Gocmen H, Uzunkulaoglu H, Karakas A, Senes M, et al. Ginkgo biloba, a free oxygen radical scavenger, affects inflammatory mediators to diminish the occurrence of experimental myringosclerosis. Acta Otolaryngol 2008;17:1-6.

[31] Diamond BJ, Shiflett SC, Feiwel N, Matheis RJ, Noskin O, Richards JA, et al. Ginkgo biloba extract: mechanisms and clinical indications. Arch Phys Med Rehabil 2000;81:668-78.

[32] Gryglewski RJ, Korbut R, Robak J, Sweiss J. On the mechanism of antithrombotic action of flavonoids. Biochem Pharmacol 1987;36:317-22.

[33] Kudolo GE, Dorsey S, Blodgett J. Effect of ingestion of Ginkgo biloba extract on platelet aggregation and urinary prostanoid excretion in healthy and type 2 diabetic subjects. Thromb Res 2003;108:151-60.

[34] Robak J, Gryglewski RJ. Flavonoids are scavengers of superoxide anions. Biochem Pharmacol 1988:37:837-41.

[35] Maitra I, Marcocci L, Droy-Lefaix MT, Packer L. Peroxyl radical scavenging activity of Ginkgo biloba extract EGb 761. Biochem Pharmacol 1995;49:1649-55.

[36] Oyama Y, Chikahisa L, Ueha T, Kanemaru K, Noda K. Ginkgo biloba extract protects brain neurons against oxidative stress induced by hydrogen peroxide. Brain Res 1996;712:349-52.

[37] Van de Water T, Ruben RJ. Growth of the inner ear in organ culture. Ann Otol Rhinol Laryngol 1974;83:1-16.

[38] Sobkowicz HM, Loftus JM, Slapnick SM. Tissue culture of the organ of Corti. Acta Otolaryngol Suppl 1993;502:3-36.

[39] Salt AN, Gill RM, Plontke SK. Dependence of hearing changes on the dose of intratympanically applied gentamicin: a meta-analysis using mathematical simulations of clinical drug delivery protocols. Laryngoscope 2008;118: 1793-800.

[40] Day AS, Lue JH, Yang TH, Young YH. Effect of intratympanic application of aminoglycosides on click-evoked myogenic potentials in guinea pigs. Ear Hear 2007;28:18-25.

[41] Trayner ID, Rayner AP, Freeman GE, Farzaneh F. Ouantitative multiwell myeloid differentiation assay using dichlorodihydrofluorescein diacetate $\left(\mathrm{H}_{2}\right.$ DCF-DA $)$ or dihydrorhodamine $123\left(\mathrm{H}_{2} \mathrm{R} 123\right)$. J Immunol Methods 1995; $186: 275-84$.

[42] Sha SH, Taylor R, Forge A, Schacht J. Differential vulnerability of basal and apical hair cells is based on intrinsic susceptibility to free radicals. Hear Res 2001;155: $1-8$.

[43] Guthrie OW. Aminoglycoside induced ototoxicity. Toxicology 2008;249:91-6.

[44] Rybak LP, Ramkumar V. Ototoxicity. Kidney Int 2007;72:931-5.

[45] Liu HY, Chi FL, Gao WY. Taurine attenuates aminoglycoside ototoxicity by inhibiting inducible nitric oxide synthase expression in the cochlea. Neuroreport 2008:19:117-20.

[46] Heinrich UR, Selivanova O, Brieger J, Mann WJ. Endothelial nitric oxide synthase upregulation in the cochlea of the guinea pig after intratympanic gentamicin injection. Eur Arch Otorhinolaryngol 2006;263:62-8.

[47] Yoshikawa T, Naito Y, Kondo M. Ginkgo biloba leaf extract: review of biological actions and clinical applications. Antioxid Redox Signal 1999;1:469-80.

[48] Hyun SK, Jung HA, Chung HY, Choi JS. In vitro peroxynitrite scavenging activity of 6-hydroxykynurenic acid and other flavonoids from Ginkgo biloba yellow leaves. Arch Pharm Res 2006;29:1074-9.

[49] Kang JW, Kim JD, Song K, Kim SH, Yoon JH, Kim KS. Kaempferol and quercetin, components of Ginkgo biloba extract (EGb 761), induce caspase-3-dependent apoptosis in oral cavity cancer cells. Phytother Res 2010;24 Suppl 1:577-82.

[50] Chang TK, Chen J, Teng XW. Distinct role of bilobalide and ginkgolide A in the modulation of rat CYP2B1 and CYP3A23 gene expression by Ginkgo biloba extract in cultured hepatocytes. Drug Metab Dispos 2006;34:234-42.

[51] Rajaraman G, Chen J, Chang TK. Ginkgolide A contributes to the potentiation of acetaminophen toxicity by Ginkgo biloba extract in primary cultures of rat hepatocytes. Toxicol Appl Pharmacol 2006;217:225-33.

[52] Shi C, Zhao L, Zhu B, Li Q, Yew DT, Yao Z, et al. Protective effects of Ginkgo biloba extract (EGb761) and its constituents quercetin and ginkgolide $B$ against $\beta$-amyloid peptide-induced toxicity in SH-SY5Y cells. Chem Biol Interact 2009;181:115-23. 